Como citar: Schott, F., Vidal, J., Pasche, A., \& Corrêa, G. (2021). Transtorno depressivo maior: diferentes possibilidades para pacientes resistentes ao tratamento. PSI UNISC, 5(2), 125-141. doi:

10.17058/psiunisc.v5i2.15284

\title{
Transtorno depressivo maior: diferentes possibilidades para pacientes resistentes ao tratamento
}

\author{
Transtorno depresivo mayor: diferentes posibilidades para pacientes resistentes al \\ tratamiento
}

\section{Major depressive disorder: different possibilities for treatment-resistant patients} \author{
Universidade Federal de Santa Maria (UFSM), Santa Maria - RS/Brasil \\ ORCID: 0000-0003-3283-2218 \\ E-mail: psicologafabiane.schott@gmail.com
}

Júlia Luciane Vidal

Universidade Federal de Santa Maria (UFSM), Santa Maria - RS/Brasil ORCID: 0000-0003-1079-5751

E-mail: juhlvidal@gmail.com

\author{
Alice Dias Pasche \\ Universidade Federal de Santa Maria (UFSM), Santa Maria - RS/Brasil \\ ORCID: 0000-0003-2829-2052 \\ E-mail: alicepasche@ hotmail.com
}

Guilherme Corrêa

Universidade Federal de Santa Maria (UFSM), Santa Maria - RS/Brasil ORCID: 0000-0002-0897-477X

E-mail: guilherme.corrêa@fisma.com

\begin{abstract}
Resumo
O transtorno depressivo maior é considerado atualmente uma questão de saúde pública devido à alta taxa de prevalência na população em geral. Com frequência, esse transtorno não é diagnosticado nem tratado, gerando impactos relevantes em todas as esferas da vida do indivíduo. Objetivando analisar o que se tem discutido sobre o assunto realizou-se uma revisão narrativa acerca dos diferentes recursos envolvidos no tratamento do transtorno depressivo maior para pacientes resistentes. Os resultados encontrados indicaram que a depressão é facilmente confundida com outras patologias justamente pelos sintomas físicos que os pacientes apresentam. O estigma que envolve a saúde mental também é um fator agravante ao tratamento. Por meio dessa revisão foi possível constatar que embora existam tratamentos médicos promissores e eficientes, como a eletroconvulsoterapia e a estimulação magnética transcraniana, eles não são acessíveis à toda população, uma vez que para esses procedimentos não há investimento no Sistema Único de Saúde. Já a psicoterapia cognitivo-comportamental combinada ao uso de fármacos sobressai-se em relação a outros tipos de recursos disponíveis, principalmente nos aspectos que envolvem qualidade de vida e funcionalidade.
\end{abstract}

Palavras-chaves: Transtorno depressivo maior; Tratamentos; Terapia cognitivo-comportamental.

\section{Resumen}

El trastorno depresivo mayor se considera actualmente un problema de salud pública debido a la alta tasa de prevalencia en la población general. A menudo, este trastorno no se diagnostica ni se trata, provocando impactos relevantes en todos los ámbitos de la vida del individuo. Con el fin de analizar lo discutido sobre el tema, se realizó una revisión narrativa sobre los diferentes recursos involucrados en el tratamiento del trastorno depresivo mayor para pacientes resistentes. Los resultados encontrados indicaron que la depresión se confunde fácilmente con otras patologías precisamente por los síntomas físicos que presentan los pacientes. El estigma que rodea a la salud mental también es un factor 
agravante para el tratamiento. A través de esta revisión se pudo constatar que si bien existen tratamientos médicos prometedores y eficientes, como la terapia electroconvulsiva y la estimulación magnética transcraneal, no son accesibles para toda la población, ya que no hay inversión en el Sistema Único de Salud para estos procedimientos. La psicoterapia cognitivoconductual combinada con el uso de drogas destaca en relación con otro tipo de recursos disponibles, principalmente en aspectos que involucran calidad de vida y funcionalidad.

Palabras clave: Trastorno depresivo mayor; Tratamento; Terapia cognitiva-comportamiento - táctico.

\begin{abstract}
Major depressive disorder is currently considered a public health issue due to the high prevalence rate in the general population. Often, this disorder is neither diagnosed nor treated, causing relevant impacts in all spheres of the individual's life. In order to analyze what
\end{abstract}

has been discussed on the subject, a narrative review was carried out about the different resources involved in the treatment of major depressive disorder for resistant patients. The results found indicated that depression is easily confused with other pathologies precisely because of the physical symptoms presented by the patients. The stigma surrounding mental health is also an aggravating factor for treatment. Through this review, it was possible to verify that although there are promising and efficient medical treatments, such as electroconvulsive therapy and transcranial magnetic stimulation, they are not accessible to the entire population, since there is no investment for those procedures in the Unified Health System. Cognitive-behavioral psychotherapy combined with the use of drugs stands out in relation to other types of available resources, mainly in aspects that involve quality of life and functionality.

Keywords: Major depressive disorder; Treatments; Cognitive behavioral therapy.

\section{Introdução}

A palavra depressão tem origem do latim depressus, cujo significado refere-se ao ato de deprimir-se. A depressão é considerada um transtorno mental, decorrente da interação entre diversos fatores. Estima-se que 350 milhões de pessoas sofrem com esse transtorno a nível mundial, sendo o estresse e a solidão dois elementos destacados quando fala - se nesse assunto (World Health Organization [WHO], 2016). Esse número corresponde a 5\% da população geral que manifesta os sintomas da doença. Para Bolsoni-Silva e Loureiro (2015) no Brasil, um a cada sete adultos é afetado, sendo estes dados variáveis de acordo com a região pesquisada. Esse índice pode ser mais elevado em pessoas que tenham outras doenças como comorbidades, como por exemplo o câncer, podendo chegar a um percentual de 47\%. Estudos longitudinais demonstraram que a depressão pode ser crônica e recorrente e que as taxas de remissão podem variar entre 9\% a 80\% (Cardoso, 2011).

\section{O Transtorno Depressivo Maior (TDM)} tornou-se uma questão de saúde pública, resultando da cultura da produtividade e outros fatores associados, cujo início muitas vezes ocorre na infância ou adolescência, estendendo-se e atingindo inúmeras pessoas das demais faixas etárias (Bahls \& Bahls, 2003). O TDM pode surgir em qualquer idade, porém as chances aumentam no início da adolescência. Não obstante, a Organização mundial da Saúde (OMS) aponta o TDM como a maior causa de adoecimento em adolescentes ao redor do mundo (World Health Organization, 2013). De acordo com Bahls e Bahls (2003) o TDM em crianças e adolescentes no Brasil aponta índices de 0,4 a $3,0 \%$, em crianças, e de 3,3 a $12,4 \%$ em adolescentes.

O transtorno depressivo maior surge na ausência ou deficiência de vieses cognitivos positivos. As pessoas com depressão não conseguem imaginar o futuro de outra maneira, e sim somente baseada em seu estado atual (Hofmann, 2014). O indivíduo acometido manifesta ansiedade por meio do medo intenso, sem que haja uma razão objetiva, exprimindo no conteúdo dos pensamentos pessimismo, inutilidade, desesperança, autodepreciação, embotamento afetivo, diminuição da atenção, dificuldade de concentração, déficit de memória, capacidade de comunicação reduzida, ideias de culpa e até mesmo de suicídio (Andrade \& Mello, 2010).

A classificação dos sintomas do TDM pode ser realizada em três níveis de acordo com 
a Classificação estatística internacional de doenças e problemas relacionados à saúde (CID-10; WHO, 2010): leve, moderado e grave, conforme o número de sintomas apresentados, e sua intensidade. Os critérios diagnósticos conforme a CID10 para o episódio depressivo apresenta-se da seguinte forma: dois dos três sintomas principais (humor deprimido, perda de interesse ou prazer e energia reduzida), podendo ser acompanhados de outros sintomas: concentração e atenção, assim como autoestima e autoconfiança, reduzidas, aliadas à prejuízo funcional ou social (WHO, 2010).

Os fatores de risco para a depressão conforme o DSM-5 aponta, envolvem os seguintes fatores: histórico familiar de depressão, doença física, episódio anterior de depressão, acontecimentos estressantes ou perdas e abuso de medicamentos ou drogas, problemas interpessoais, ou mudanças, como divórcio, desemprego, perda de um ente querido são muito comuns no quadro de depressão (American Psychiatry Association, 2014). Portanto compreender o contexto social e interpessoal é essencial para elaborar um plano de tratamento e prevenir episódios futuros (Hofmann, 2014).

Em relação ao gênero, entre homens e mulheres, o sexo feminino prevalece mostrando-se mais afetado, na proporção de 2:1 entre pessoas com transtorno depressivo maior (TDM), no entanto alguns estudos questionam tal fato. Um estudo apresentando no XXIII Encontro Anual da Sociedade de Medicina Comportamental nos EUA demonstrou que homens são menos propensos a relatar sintomas depressivos quando questionados sobre depressão, e referem a um número mais expressivo de sintomas depressivos quando questionados sobre o bem-estar (Goodier, 2014).

Embora o acesso aos serviços de saúde e a busca por ajuda tenha expandido nas últimas décadas, o alcance da população aos diferentes tratamentos consiste ainda em um grave problema. Conforme um estudo populacional realizado no Brasil, 7,6\% (IC 95\% CI 7.2-8.1) das pessoas entrevistadas apresentavam diagnóstico prévio de depressão realizado por médico ou profissional de saúde, representando um total de 11.179 adultos (Stopa et al., 2015). O predomínio de manifestações de queixas físicas dificulta o diagnóstico preciso, uma vez que os pacientes quando atendidos na atenção primária relatam sintomas fisiológicos em vez de queixas emocionais, o que pode mascarar o transtorno. Esse evento justifica o fato de $50 \%$ a $60 \%$ dos casos de TDM não serem detectados na atenção básica, havendo altos índices de recorrência, cerca de 50 a $90 \%$ dos casos (Carneiro \& Dobson, 2016), com taxas de suicídio equivalentes a $60 \%$ da totalidade dos casos (World Health Organization, 2016).

Como forma de tratamento, os fármacos comumente utilizados e têm por finalidade a remissão dos sintomas da depressão, são os tricíclicos, os inibidores seletivos da recaptação da serotonina, os inibidores seletivos da recaptação da noradrenalina e os inibidores da IMAO (inibidores da monoamina-oxidase), além dos antidepressivos multimodais. Todas essas substâncias elevam os níveis dos neurotransmissores monoaminérgicos no encéfalo. No entanto, sua ação terapêutica requer semanas para produzir os efeitos desejados (Bear, Connors, \& Paradiso, 2017).

Dos tratamentos disponíveis atualmente pode-se citar ainda a utilização da terapia cognitivo-comportamental, que se mostra eficiente para pacientes com depressão. Uma das principais manifestações dos acometidos está no conteúdo dos pensamentos, caracterizado por negativismo, ausência de perspectivas positivas e desesperança em relação a si, aos outros e ao futuro, contrastando com a realidade dos fatos em si (Hofmann, 2014). A TCC dispõe de técnicas capazes de auxiliar o paciente a ser seu próprio terapeuta (Beck, 2013). Sendo assim, os pacientes têm maior habilidade em reconhecer seus sintomas e, assim, prevenir recaídas, o que ocasiona também redução de gastos com o tratamento (Wiles et al., 2014). Em geral, as pesquisas demonstram a eficácia da TCC na redução de sintomas e na prevenção de recaída e recorrência no tratamento da depressão leve, 
moderada e grave (Almeida \& Lotufo Neto, 2003; Canadian Network for Mood and Anxiety Treatments, 2012; Dobson, 2016; Friedman et al., 2009; Koenig \& Thase, 2009).

Associado aos tratamentos possíveis está a eletroconvulsoterapia (ECT). Essa alternativa pode ser indicada a pacientes com transtorno depressivo grave, não aderentes à medicação. Como critérios à sua finalidade está a intensidade ou frequência e a duração dos sintomas depressivos, a presença de manifestações psicóticas ou catatônicas, o risco de suicídio ou ainda a necessidade urgente de melhora, diante de rápida deterioração da saúde física. A ECT consiste num procedimento que induz crises convulsivas por meio da passagem de uma corrente elétrica pelo cérebro com finalidade terapêutica (American Psychiatry Association, 2001).

Existe no Brasil apenas 13 instituições que oferecem a ECT de forma gratuita aos seus pacientes (Ribeiro, Melzer-Ribeiro, Rigonatti, \& Cordeiro, 2012). Houve movimentos de Secretarias da Saúde de alguns Estados solicitando o custeio da ECT pelo SUS. No entanto, não foram atendidas, sendo arquivadas nos anos de 2004 e 2005 pelo Ministério da Saúde. Outro recurso de tratamento existente é a estimulação magnética transcraniana (EMT), que produz correntes de campos eletromagnéticos induzidas no cérebro de modo não-invasivo, podendo ser suficiente para despolarizar neurônios. (Kobayashi \& PascualLeone, 2003). No Brasil a EMT foi reconhecida oficialmente pelo Conselho Federal de Medicina em 2012, porém na atualidade somente quatro Estados realizam o procedimento em instituições de ensino ou do setor privado. Este exposto já é um fator limitante, tendo em vista que está inacessível a maioria da população.

No cenário mundial, evidências de pesquisas recentes sugerem que o uso da psilocibina, princípio ativo dos cogumelos, no tratamento da doença. O componente da substância psicodélica é extraído de plantas ou sintetizadas em laboratório, e induz estados alterados de consciência. Do ponto de vista químico, elas aumentam a quantidade de neurotransmissores no cérebro, em especial a serotonina, que controla as emoções e regula o domínio sensorial, o motor e a capacidade associativa do cérebro. Elas reduzem a atividade na amígdala, parte do cérebro que regula a resposta ao medo. $\mathrm{E}$ aumenta a atividade no córtex pré-frontal, parte associada ao raciocínio, humor e percepção (Davis et al., 2020).

O efeito terapêutico estaria relacionado a maior fluidez da atividade cerebral. Com uma ou duas administrações associado ao apoio psicológico produziu efeitos antidepressivos em pacientes com câncer, e naqueles com depressão resistente ao tratamento. Em um ensaio clínico randomizado com 24 indivíduos com transtorno depressivo maior descobriu-se que a terapia assistida por psilocibina foi eficaz na produção de efeitos antidepressivos expressivos, rápidos e comprovados, em pacientes com transtorno depressivo maior (Davis et al., 2020).

Assim, a relevância deste estudo justifica-se pelo aumento significativo nas últimas décadas de sujeitos acometidos pelo transtorno depressivo maior, o que afeta negativamente a vida das pessoas, seja pessoal ou profissionalmente (World Health Organization, 2016). Segundo o Banco Mundial, no ranking dos países que apresentam alta incidência de depressão, o Brasil aparece em quinto lugar com $(5,8 \%)$. Por meio desses indicadores, pode-se afirmar que a depressão e a ansiedade têm custo de1 trilhão de dólares à economia mundial a cada ano. E cada dólar investido em tratamentos leva a um retorno de quatro dólares em termos de saúde e capacidade dos trabalhadores, ou seja, investir em saúde mental e qualidade de vida é mais produtivo do que lidar com os prejuízos causados pela negligência e o descaso do cuidado (World Health Organization, 2016). Posto isto, o presente estudo poderá promover um entendimento a respeito dos tratamentos disponíveis para a depressão.

\section{Método Delineamento}


Para o desenvolvimento desta pesquisa, foi realizada uma revisão bibliográfica narrativa. Esse tipo de estudo utiliza publicações amplas a fim de descrever e discutir o estado da arte, o desenvolvimento sobre um determinado tema, de maneira teórica ou contextual, sem necessariamente explicitar as fontes de informação aplicadas, a metodologia para busca das referências, e os critérios empregados na avaliação e seleção dos trabalhos (Vosgerau \& Romanawski, 2014). Esse Estudo permitiu atualização do conhecimento sobre uma temática específica, evidenciando novas ideias, métodos e subtemas, assim como estabelecer relações com produções anteriores, identificando temáticas recorrentes, apontando novas perspectivas.

A coleta de dados foi realizada por meio de busca nas principais plataformas online disponíveis, assim sendo: ScIELO, LILACS e Bvspsi. Optou-se por estudos em língua portuguesa disponibilizados de forma completa e que contemplavam a temática da pesquisa, e de exclusão àqueles que não abrangiam tal temática ou fugiam do assunto principal, isto é a variabilidade de tratamentos para a depressão. A busca de artigos nos periódicos foi demarcada pelos seguintes descritores combinados "depressão", "tratamento para depressão", "terapia cognitiva”, "terapia cognitivo-comportamental". O critério utilizado para inclusão das publicações foi conter expressões utilizadas nas buscas no título ou palavras-chave, ou ter explícito no resumo que o texto se relacionava à associação de tratamentos utilizados para transtorno depressivo maior. Os artigos excluídos não apresentavam o critério de inclusão estabelecido e/ou apresentavam duplicidade, ou seja, publicações recuperadas em mais de uma das bases de dados.

\section{Análise dos dados}

A análise dos dados teve o objetivo de levantar, reunir, avaliar criticamente os resultados de diversos estudos primários. Primeiramente foi realizada a leitura exploratória dos estudos encontrados. Após essa etapa foram selecionados os materiais que mais se encaixavam no estudo, em seguida realizou-se a leitura interpretativa buscando compreender os significados, estabelecendo relações entre eles, conferindo sentidos mais amplos aos resultados que foram obtidos. Após essa fase, iniciou-se a construção de sínteses e conclusões descritivas das informações (Gomes \& Caminha, 2014).

\section{Resultados}

A escolha dos artigos contemplou a temática de pesquisa, e o cruzamento entre os temas: terapia cognitivo-comportamental e depressão, e as demais categorias envolvendo outros tratamentos alternativos a pacientes depressivos. A partir da análise dos conteúdos selecionados foi possível identificar, interpretar e descrever o que os diferentes trabalhos apresentaram sobre o tema e organizá-los por assunto. Portanto, na sequência são apresentados esses achados, de acordo com o tema da pesquisa. No primeiro tópico, abordouse a eficácia da terapia cognitivocomportamental na prevenção a recaídas e o desenvolvimento de resiliência em pacientes com TDM. Em seguida, a potencialização de tratamentos combinados para transtorno depressivo maior: $2+2=6$, e por último, os tratamentos alternativos para pacientes refratários ao uso de medicação e psicoterapia.

\section{Eficácia da Terapia cognitivo- comportamental na prevenção a recaídas e desenvolvimento de resiliência em pacientes com TDM}

De acordo com Beck e colaboradores (1997), a psicoterapia, a longo prazo, traz mais benefícios que a farmacoterapia usada unicamente, uma vez que, o paciente é ativo em seu processo terapêutico. Dessa forma, por meio da psicoeducação o paciente aprende a monitorar, testar na realidade e modificar padrões estereotipados de pensamentos negativos, desenvolvendo outras maneiras de pensar e agir perante certas situações (Beck, Rush, Shaw \& Emery, 1997). 
Essas habilidades aprendidas lhe permitiriam lidar mais facilmente com novos episódios recorrentes, e, possivelmente prevenir novas depressões. A fim de que o paciente incorpore as técnicas cognitivocomportamentais ao seu repertório de habilidades de enfrentamento é recomendável que o terapeuta explique o propósito da terapia, que é breve e focado, ensinando-lhe técnicas para que este possa usá-las quando sentir necessidade. É relevante que o terapeuta seja enfático quanto a participação ativa de seu paciente no processo, e atribua a ele os progressos alcançados, estimulando a independência e a autoconfiança (Beck \& Bredemeier, 2016).

A fim de evitar recaídas no futuro o terapeuta poderá discutir abertamente com o paciente acerca de sua conceituação cognitiva. Requerendo do terapeuta investigação com certa frequência sobre o conteúdo do pensamento do paciente, na tentativa de esquematizar o seu funcionamento. Sabe-se que eventos vitais negativos têm maior chance de desencadear sintomas ou recidivas depressivas se forem relacionados com as crenças disfuncionais centrais dos pacientes (Beck \& Bredemeier, 2016).

Assim, o encerramento prematuro da terapia pode elevar a chance de recaída, quando houver alívio rápido dos sintomas após poucas sessões. Essa melhora precoce pode acontecer devido a fatores que não sejam estejam ligados diretamente à terapia cognitiva. É importante que o paciente aprenda a lidar com o possível retorno dos sintomas depressivos impedindo que evoluam para um novo episódio depressivo. Um fator emergente na literatura é a metacognição (Powell, Abreu, Oliveira, \& Sudak, 2008).

Esse conceito envolve um
funcionamento cognitivo em
pensamentos/sentimento negativos
vivenciados como eventos mentais e não como
a expressão da realidade. Assim, ao identificar
o conteúdo dos pensamentos negativos
(extremos, absolutistas e dicotomicos, em
termos de "tudo ou nada") que surgem durante

períodos de disforia e estresse, o paciente conseguiria lidar com eles de modo mais saudável e até substituí-los por outros. Desse modo, quando o indivíduo está consciente sobre o conteúdo dos seus pensamentos e como afeta o seu comportamento, ele estará mais apto a prevenir recaídas (Beck, 2013).

Adicional ao processo de prevenção a recaídas está o tratamento dos sintomas depressivos residuais. Esse enfoque baseia-se na hipótese de que os sintomas residuais e do TDM são similares, uma vez que os padrões da recaída depressiva tendem a ser semelhantes ao episódio inicial. A ansiedade e a irritabilidade têm se mostrado como importantes alvos desse tipo de abordagem preventiva (Fava, Rafanelli, Grandi, Conti, \& Belluardo, 1998).

\section{Potencialização de tratamentos combinados para transtorno depressivo maior: $2+2=6$}

O TDM pode ocorrer em diversos quadros clínicos, tais como: demência, esquizofrenia, alcoolismo, doenças clínicas etc. A manifestação da depressão pode estar relacionada a situações estressantes ou a circunstâncias sociais e econômicas adversas. Como síndrome, a depressão inclui não apenas alterações do humor (tristeza, irritabilidade, incapacidade de sentir prazer, apatia), mas também vários outros aspectos, incluindo alterações cognitivas, psicomotoras e vegetativas (sono, apetite etc.) (Tomas \& Carvalho, 2014).

A psicoterapia é fundamental em todos os casos de TDM, a partir do momento em que houver uma redução na frequência de certas atividades mantidas anteriormente pelo indivíduo, tidas como prazerosas e aumento, concomitante, comportamentos de fuga e esquiva de situações adversas. Tais comportamentos estariam relacionados à depressão em razão da dificuldade do indivíduo em acessar os reforçadores disponíveis em seu ambiente (Carneiro \& Dobson, 2016). A redução na frequência de emissão de comportamentos emitidos por reforçadores positivos poderia ser em decorrência do custo 
de resposta exigido em certas contingências de reforçamento e por mudanças repentinas no ambiente em que o indivíduo está inserido (Abreu \& Santos, 2008).

Em diagnósticos que apontam para o risco de vida ao paciente, reforça-se a importância do atendimento psicológico. Os sujeitos que apresentam sintomas psicóticos possuem risco de suicídio ainda maior (American Psychiatry Association, 2014; Rothschild, 2013; Gottlieb, Mueser, Rosenberg, Xie, \& Wolfe, 2011; World Health Organization, 2009). O terapeuta deve estar atento e avaliar se o risco está presente ao paciente. Torna-se necessário investigar tanto a existência de ideações e comportamentos suicidas. Nesse sentido, deve-se considerar que a depressão é um transtorno multifacetado, e por isso, não existe um tipo único de protocolo psicoterápico (ou farmacológico) que funcionará em todos os pacientes ou países (Dobson, 2016).

Quanto ao uso do tratamento medicamentoso, houve uma mudança nas necessidades e objetivos do tratamento para depressão desde que se iniciaram avaliações sistemáticas de resultados em estudos clínicos de antidepressivos nos anos 1970. Inicialmente, a preocupação era voltada para a resposta clínica, que era importante pois indicava que paciente e médico reconheciam que havia uma melhora relevante. A resposta clínica nesses casos era verificada por meio da redução dos sintomas em uma escala de classificação de depressão como a Escala de Classificação de Depressão de Montgomery-Åsberg (MADRS) ou a Escala de Classificação de Depressão de Hamilton [HAM-D] (Mclntyre, 2015).

Contudo, posteriormente identificou-se que ter apenas uma resposta clínica não bastava, assim como a remissão do sintoma era importante. A constatação veio por meio de evidências que indicavam que sintomas residuais de pacientes que não estavam em remissão, levavam a desfechos piores, no ideação e comportamento suicida. Normalmente media-se ou definia a remissão como um escore dentro da variação normal numa escala de sintomas como MADRS ou HAM-D (Mclntyre, 2015).

No entanto, conforme Mclntyre (2015) afirma, atualmente sabe-se que a remissão não deve ser a meta final. Hoje a investigação aponta que os pacientes alcançam melhores resultados, ou seja, atingem a recuperação funcional. Essa recuperação inclui o retorno às suas vidas sociais, seus empregos, e a ter funcionamento pré-morbidez, o que significa que estão bem, como antes do tratamento e da depressão iniciar. É este o objetivo do tratamento farmacológico: tornar mais funcional a vida do paciente com depressão.

Esses objetivos são relevantes para a vida dos pacientes, sobretudo para sua qualidade de vida em geral. A realização do tratamento medicamentoso da depressão é muito heterogênea e engloba um grupo muito grande de distúrbios dentro da área de TDM, incluindo certas características distintas para certos grupos e não para outros (Mclntyre, 2015). A fim de obter resultados concretos nos pacientes, deve-se buscar por domínios mais específicos dentro deste grupo heterogêneo de TDM. Alguns destes domínios incluem o humor especificamente, assim como sono, a disfunção sexual, e a cognição. Pressupõe-se nessa abordagem de tratamento, que seria possível maximizar os resultados desse recurso medicamentoso em geral.

$$
\begin{aligned}
& \text { Os mecanismos de ação da psicoterapia } \\
& \text { e } \quad \operatorname{dos} \text { fármacos assemelham-se }
\end{aligned}
$$
neurobiologicamente, pois ambos podem alterar os circuitos neuronais. Dessa forma, os dois recursos demonstram ser efetivos no tratamento de transtornos psiquiátricos ou que sua associação seja terapeuticamente sinérgica (potencializada). Na psicoterapia esse efeito é alcançado pela hipótese de indução nas alterações epigenéticas dos circuitos cerebrais, o que possivelmente elevaria à eficiência do processamento de informações nos neurônios disfuncionais, melhorando os sintomas dos transtornos psiquiátricos, exatamente como os fármacos (Stahl, 2014) 
Em vista disso, a psicoterapia pode ser compreendida como fármacos epigenéticos, com finalidade de agentes terapêuticos que atuam epigeneticamente, de modo semelhante ou complementar aos fármacos. $\mathrm{O}$ processamento deficiente de informações em circuitos específicos está intimamente ligado a sintomas psiquiátricos específicos. Não somente os genes, mas também as experiências ambientais, o estresse, a aprendizagem, a psicoterapia e os psicofármacos modificam os sistemas neurotransmissores e alteraram a atividade desses circuitos, para criar ou aliviar sintomas psiquiátricos e potencializar a eficiência do processamento de informações nesses circuitos (Stahl, 2014).

Portanto, experiências positivas e negativas podem impulsionar a produção de alterações epigenéticas na expressão gênica, e, com efeito, alterações epigenéticas na transcrição de genes parecem estar subjacentes às memórias a longo prazo, sejam elas boas ou ruins. As memórias ruins de traumas na infância podem desencadear transtornos psiquiátricos ao produzirem alterações desfavoráveis nos circuitos cerebrais. Já as boas memórias formadas durante a psicoterapia podem alterar favoravelmente os mesmos circuitos cerebrais que servem de alvo para os fármacos e, de modo semelhante, potencializar a eficiência do processamento de informações e, assim, aliviar os sintomas (Stahl, 2014).

Se tanto os psicofármacos quanto a psicoterapia convergem para circuitos cerebrais sua combinação pode ser aproveitada a fim de aumentar a eficácia e obter melhores resultados em pacientes com transtornos psiquiátricos. Pode-se levantar a hipótese de que, quando bem-sucedida, essa abordagem forma uma sinapse da nova perspectiva do "advogado de defesa interno" para combater e inibir o circuito que intermedia a ativação da primeira aprendizagem, isto é, a crença nuclear distorcida do "promotor interno". A combinação da psicoterapia com antidepressivos tem o potencial de tornar os resultados maiores do que a soma das partes, ou seja, $1+1=3$, o fenômeno do sinergismo terapêutico (Stahl, 2014).

\section{Tratamentos alternativos para pacientes refratários ao uso de medicação e psicoterapia}

Dos tratamentos existentes hoje para pacientes com TDM que não aderem ao uso de medicação e /ou psicoterapia pode-se citar a eletroconvulsoterapia (ECT) e a estimulação magnética transcraniana (EMT). A decisão pela escolha e tratamento com a utilização da ECT depende de vários fatores, incluindo a gravidade e cronicidade da depressão do paciente. Embora a ECT seja altamente eficaz, seu uso é normalmente reservado para pacientes refratários a antidepressivos. Porém esta pode ser indicação terapêutica de primeira escolha em pacientes com síndromes psiquiátricas agudas como mania delirante, catatonia maligna e síndrome maligna dos neurolépticos e em caso de sintomas psicóticos graves e com alto risco de suicídio (Pastore, Bruno, Nardi, \& Dias, 2008).

Mesmo sendo considerada uma técnica de segunda escolha (após a falha do tratamento farmacológico), o uso da ECT não deve ficar restrito a esta condição (Moser, Lobato, \& Belmonte-De-Abreu, 2005). Alguns critérios devem ser levados em consideração em relação a essa técnica, tais como; o perfil do paciente (sexo, diagnóstico e idade), o tempo de indução da convulsão, a quantidade de aplicações necessárias, e deve-se avaliar os riscos clínicos decorrentes de outras doenças, os quais podem levar a complicações e a morte (Pastore et al., 2008).

Acerca da eficácia e do mecanismo dessa técnica existem algumas hipóteses, uma delas é que ocorra um aumento expressivo de prolactina após a aplicação, consequentemente diminuindo os sintomas depressivos. A ECT e a Estimulação magnética transcraniana (EMT) são tratamentos "físicos", não farmacológicos, mas, que apresentam diferenças em sua administração tanto do ponto de vista do médico como do paciente. As diferenças entre as duas técnicas estão evidenciadas a seguir.

A EMT e ECT assemelham-se no sentido de que ambas alteram a atividade 
neuronal e modificam o humor. No entanto, algumas questões sobre o tratamento com a EMT na depressão ainda precisam ser melhor analisadas. Em que fase do TDM usar, de que forma usar, adjunta aos antidepressivos, potencializando seus efeitos ou como primeira opção. Ou ainda após o uso da ECT sem sucesso. Essas dúvidas e respostas não foram elucidadas. Muitos estudos foram realizados com pacientes com depressão maior unipolar resistentes a múltiplas intervenções farmacológicas. O nível de resistência pode ter impacto na resposta da EMT no tratamento da depressão. É preciso reconsiderar o momento ideal de utilizar a EMT como recurso terapêutico (Lefaucheur et al., 2014).

Em relação a outros tratamentos, não houve estudos brasileiros sobre o tema. No entanto cabe destacar que os resultados sobre a utilização da psilocibina no tratamento da depressão ainda são iniciais, limitados a um pequeno grupo de pacientes que não responderam a outros tratamentos. Mesmo assim, os resultados da terapia experimental são surpreendentes. Um ensaio clínico randomizado, publicado na JAMA Psychiatry, apresentou os efeitos antidepressivos rápidos e duradouros da terapia assistida por psilocibina entre pacientes com TDM. $\mathrm{O}$ estudo atual mostrou que a resposta antidepressiva foi clinicamente significativa à terapia com psilocibina. O efeito benéfico se manteve após 4 semanas, com $71 \%$ dos participantes continuando a mostrar uma resposta clinicamente significativa ( $\geq 50 \%$ de redução na pontuação da escala Hamilton que avalia sintomas depressivos [HAM-D] na quarta semana de acompanhamento. Descobriu-se ainda, que a psilocibina tem baixo potencial para dependência e efeitos colaterais mínimos. A descoberta fornece uma nova visão sobre um possível novo tratamento para o TDM (Davis et al., 2020).

\section{Discussões}

O trabalho realizado levou em consideração os principais temas que dizem respeito ao tratamento para depressão dentro das diversas áreas: médicas, farmacológicas e psicológicas, embora o interesse estivesse voltado também no sentido de identificar outros possíveis tratamentos. Em relação aos artigos analisados pela autora, foi possível avaliar que atualmente acerca dos tratamentos para TDM, a utilização de ECT e ETM ainda são técnicas pouco conhecidas pela maioria da população que poderia se beneficiar com esse recurso, pois no caso da ECT a aplicação é restrita ao âmbito hospitalar, uma vez que se utiliza de sedativos para o procedimento (Freire, 2016).

Os fatores negativos que cercam a utilização da ECT ainda persistem, embora essa terapêutica apresente eficiência comprovada, principalmente quando usada para casos de TDM grave, psicose e risco elevado para o suicídio. Entretanto, os efeitos colaterais cognitivos são a maior limitação da ECT, ao diminuírem a satisfação do paciente e contribuindo com o estigma associado ao tratamento. Dos efeitos cognitivos, o déficit na memória é o mais importante. Logo após o curso da ECT, a maioria dos pacientes manifesta dificuldade em reter informações recém-aprendidas (amnésia anterógrada) e lembrar de eventos que ocorreram semanas ou meses antes da ECT (amnésia retrógrada) (Freire, 2016). Existe ainda uma ausência de consenso sobre o seu uso e precariedade de investimento pelo Ministério da Saúde conforme menciona Moser et al., (2005). Esses seriam fatores de resistência acerca do método, todavia não foram encontrados trabalhos científicos que questionassem a eficácia da ECT. Sobre o uso de ECT um estudo realizado por Freire (2016), aponta evidências satisfatórias em pacientes que usaram medicamentos durante e após o tratamento com essa técnica, todavia não há atualmente conhecimento sobre o mecanismo da melhoria sintomatológica da ECT (Freire, 2016).

Sobre a EMT há estudos que indicam uma eficácia superior à ECT quando usada para TDM leve a moderada. Essa técnica (EMT) é considerada confiável, segura e de baixo risco para pesquisa em seres humanos, sendo usada para a modulação não-invasiva de regiões corticais. Em decorrência, a EMT tem sido sugerida para o tratamento de diversas doenças 
neurológicas e psiquiátricas, reabilitação pósacidente vascular cerebral (AVC) e ainda para a aceleração do aprendizado. Apresenta poucos efeitos colaterais, é indolor e seu procedimento é realizado com o paciente consciente.

A respeito da EMT as pesquisas ressalvam que esse método requer mais estudos sobre sua continuidade terapêutica, estudos de associação com antidepressivos e perspectivas sobre determinados públicos-alvo, como o caso do uso em crianças, em gestantes ou idosos são lacunas que permanecem abertas para investigação. Perante tais exposições é notório que ambas as técnicas EMT e ECT têm mostrado um efeito significativo na neurogênese e na modulação da plasticidade cerebral, colaborando para uma mudança de paradigma do foco bioquímico (medicamentoso) para o biofísico e também como instrumento terapêutico. No entanto, os entraves consistem na entrada dos usuários ao sistema que carece de infraestrutura e investimento adequado para atender esta demanda.

Porém há concordância entre autores (Brunoni, 2012; Moffa, 2016) de que essa técnica precisa apresentar mais ensaios clínicos, precisa de mais difusão e pesquisa. Tanto na ECT como na EMT autores concordam que ambas as técnicas necessitam de novos estudos sobre sua continuação, e investigação precisa, buscando marcadores biológicos determinantes, sobre o uso associado a outros recursos, na possibilidade de haver prognóstico terapêutico eficaz (Ribeiro et al., 2012).

Cabe ressaltar que o público alvo para aplicação de ambas as técnicas em caso de TDM seriam indivíduos que por algum motivo não aderiram à psicoterapia ou à psicofármacos, ou tratamento concomitante do dueto, e também nos quadros que não houve melhora. As pessoas idosas também podem se beneficiar desse tratamento, pois acabam esquecendo ou mesmo não aderindo aos remédios, devido aos efeitos colaterais desagradáveis, da interação entre as drogas, o que torna sua aceitabilidade e tolerabilidade difíceis. Assim como as pessoas com risco elevado de suicídio e gestantes se beneficiariam. Todavia, acerca dos estudos com EMT não há pesquisas voltadas sobre o uso em gestantes e crianças, ou seja, que contemplem esse público específico (Montenegro \& Cantilino, 2016).

Um dado importante apresentado pelo DATASUS (2012), é que entre 1996 e 2012, o suicídio cresceu $154 \%$ nessa faixa etária (idosos) no Brasil. No entanto, conforme destaca Solomon (2014), o suicídio não é um fator inerente específico da depressão. Ou seja, é um problema que coexiste à depressão, e que pode ser simultâneo ao transtorno, mas não necessariamente devido a ele, pois uma pessoa com câncer pode cometer suicídio e não ter depressão (Solomon, 2014). O risco de suicídio deve ser avaliado e acompanhado pelos profissionais de saúde mental. Há estudos epidemiológicos que revelam uma redução da frequiência de suicídio com a prescrição de antidepressivos. E outros, que sugerem que o tratamento com ISRS pode elevar o risco de suicídio em alguns pacientes, principalmente no início do tratamento. Esse risco é maior em crianças e adolescentes (Cooper et al., 2014). Para tanto, alguns hospitais já utilizam do dispositivo da alta assistida no acompanhamento dos pacientes com transtornos mentais, onde é realizado o monitoramento da sintomatologia, promovendo adesão ao tratamento, visando prevenir recaídas e verificando se há o risco de suicídio. Tais práticas se inserem no contexto atual da atenção à saúde mental.

Quanto a utilização dos antidepressivos, há evidências de melhora entre $60 \%$ e $70 \%$ dos pacientes medicados, enquanto a taxa de placebo é de $30 \%$ e os ensaios clínicos não demonstram uma maior eficácia entre os medicamentos, o que não significa que cada paciente responda da mesma forma a cada medicamento (Fleck et al., 2009). Em casos de depressão maior os antidepressivos demonstram-se mais eficazes no período de crise e também na manutenção do tratamento, porém a recaída ocorre com frequência. E, estima-se que $50 \%$ dos pacientes com 
depressão não alcançam a remissão após semanas de uso de antidepressivos e que $20 \%$ desses pacientes não respondem às diversas opções farmacológicas sendo considerados refratários aos tratamentos. Já em casos de TDM leve ou moderado esse índice não apresenta resultados superiores ao placebo, quando usados de modo isolado (Fleck et al., 2009).

Já a TCC mostrou eficácia superior de $54,8 \%$, com intervalo de confiança de $95 \%$, em comparação ao uso somente de medicamento. $\mathrm{Ou}$ seja, esses dados sinalizam, que a psicoterapia por si só se sobressai em relação ao uso de medicamentos. Essas evidências vieram de um ensaio clínico randomizado realizado com 334 pacientes, na faixa etária de 12 a 18 anos com diagnóstico primário de transtorno depressivo maior não respondentes a um tratamento inicial de 2 meses com um ISRS (Brent et al., 2008).

Os caminhos para compreensão e o tratamento do episódio depressivo ainda não são conclusos, mas sem dúvidas, a psicoterapia é fundamental para o paciente. Entre as técnicas psicoterápicas, as baseadas em TCC são aquelas que despontam nas evidências científicas (Cuijpers, 2016). Entende-se que a TCC por ser uma abordagem estruturada, focal, diretiva e ativa, apresenta eficácia no tratamento de diversos transtornos psiquiátricos. Outro fator relevante da TCC, é a forma de intervenção, que é focada na maneira como o paciente interpreta cada situação. O objetivo da TCC é auxiliar o paciente a realizar uma avaliação realista da situação, visando modificar as crenças. A função do terapeuta é psicoeducar o paciente, apresentando-lhe o modelo cognitivo, destacando as interpretações e distorções cognitivas, e como essas associam-se com as emoções e o comportamento. (Wenzel, Brown, \& Beck, 2010). Na depressão, o psicoterapeuta busca mudar a visão negativa que o paciente tem de si mesmo. (Bear et al., 2017).

As evidências de eficácia da TCC também são destacadas em outros estudos. Dunn e colaboradores (2012), pesquisaram pacientes acometidos por TDM e que foram submetidos a TCC. Os participantes da pesquisa apresentaram uma melhora significativa no funcionamento psicossocial, nas fases pré e pós-tratamento da depressão, além de benefícios à curto prazo, como redução dos sintomas depressivos durante o tratamento com a TCC na fase aguda. Da mesma forma, outro estudo evidenciou que houve redução nos escores dos inventários de Beck, na repetição e na intensidade dos pensamentos automáticos negativos, bem como flexibilização de crenças nucleares e remissão dos sintomas depressivos. $\mathrm{O}$ que promove um funcionamento biopsicossocial mais satisfatório para o paciente (Matos \& Oliveira, 2013).

Há indicativos que associam déficits de habilidades sociais de assertividade (HS) em pacientes com TDM (Oliveira, 2010). As habilidades sociais referem-se a um repertório de comportamento que permite interações interpessoais bem-sucedidas. Todavia, em pacientes deprimidos, há um comprometimento destas interações. $\mathrm{O}$ conceito de comportamento assertivo envolve a afirmação dos próprios direitos e a expressão de pensamentos, sentimentos e crenças de maneira direta, honesta e apropriada; que não viole o direito de outras pessoas. A assertividade está relacionada à produção de uma imagem positiva de si mesmo e de sentimentos de respeito, enquanto que a ausência da assertividade relaciona-se a uma imagem negativa de si, e a sentimentos de pena, irritação e desprezo (Fernandes, Falcone, \& Sardinha, 2012). Por meio da TCC é possível aprimorar tais habilidades, para que assim o paciente possa lidar de maneira satisfatória com situações estressoras e/ou de vulnerabilidade, sendo um recurso que pode evitar recaídas (Campos, Del Prette, \& Del Prette, 2014).

No âmbito da saúde pública, a atividade física apresenta-se como uma forma de tratamento acessível, barata, nãofarmacológica e capaz de gerar benefícios que excedem os efeitos antidepressivos, uma vez que promovem um plano complementar à sua saúde mental e física. $\mathrm{O}$ exercício físico promove a liberação de endorfina, responsável 
pela sensação de bem-estar, melhora a tensão muscular, que, por vezes é responsável por insônia, reduz o nível de cortisol, de ansiedade e agitação e aumenta a temperatura do corpo promovendo o relaxamento. Sendo assim, é importante que os profissionais de saúde estejam atentos e preparados para identificar e conduzir de maneira correta o tratamento dos pacientes com depressão (Costa, Soares, $\&$ Teixeira, 2007).

\section{Considerações finais}

Com relação aos objetivos desta revisão, foi possível identificar que do mesmo modo que o diagnóstico ocorre de forma diferente, o desenrolar interventivo também tem suas características singulares. Alguns aspectos precisam ser levados em consideração quando fala-se em pacientes refratários ao uso de medicação e psicoterapia. Uma questão é verificar se as opções de tratamento estão disponíveis, outra é considerar riscos e benefícios de tais procedimentos. Outros fatores igualmente importantes devem ser considerados quando da escolha terapêutica, como a faixa etária dos pacientes, nível socioeconômico, histórico familiar pregresso e o gênero.

É importante ressaltar que cada tipo de estudo relacionado aos tratamentos possui uma finalidade específica, que não corresponde necessariamente a uma hierarquização de qualidade e sim ao critério de aderência por parte dos pacientes. A respeito do uso da ECT e da EMT, as dificuldades consistem sobre o alcance desse público (resistentes a tratamentos com medicamentos e psicoterapia) a esse tratamento. Logo, isso ocorre pelo fato de não haver financiamento pelo Ministério da Saúde. Por esse motivo, os serviços públicos de ECT encontram-se somente nas universidades, as quais possuem seus próprios orçamentos para custear por determinados procedimentos médicos.

Relativo ao trabalho dos psicólogos, este ainda é discreto em setores os quais a população mais se beneficiaria, como no SUS. Na realidade houve uma transformação do SUS no que diz respeito a prática dos psicólogos nesse setor, no entanto há poucos profissionais trabalhando neste contexto acessível a toda população. Este cenário reforça a importância da ampliação de políticas públicas e de ações no trabalho dos psicólogos na saúde mental, ampliando não somente a clínica, mas também as intervenções extra clínicas visando a prevenção. Com mais profissionais da psicologia no SUS, um número maior de pessoas seria beneficiado de psicoterapia tanto individual quanto em grupo, bem como seria possível acompanhar o desfecho terapêutico dos casos de TDM.

Embora a escolha do método terapêutico é referente a cada caso particular, os resultados são enfáticos ao afirmar que na atualidade enquanto recurso terapêutico mais indicado em todos os casos é a psicoterapia (pois não possui contraindicações e nem riscos inerentes) através da abordagem teórica da teoria cognitivo-comportamental, fazendo-se necessário em alguns casos o uso de farmacoterapia aliada, tendo em vista a eficácia aumentada destes recursos quando usados em conjunto. A abordagem psicoterapêutica inclusive, conforme os resultados obtidos reduz a chance de recaídas, além de auxiliar o paciente a tornar-se mais resiliente frente a novos episódios.

Por fim, foi possível identificar que para os pacientes resistentes ao tratamento, os quais se beneficiariam com outros recursos eficazes para TDM não encontram logística acessível a esses tratamentos, permanecendo inalcançáveis pela maioria da população. $O$ tratamento psicológico também não é alcançado por todos, em razão das diversas implicações oriundas de situações socioeconômicas e da não adesão ao tratamento, e ainda pela falta de recursos e investimentos na área da saúde mental para que essa forma de tratamento seja viabilizada para atender a população.

Este estudo possibilitou uma atualização acerca dos principais recursos terapêuticos existentes para pacientes refratários ao uso de medicamentos e psicoterapia. Constatou-se que permanecem 
lacunas acerca dos tratamentos, no sentido de ausências de evidências clínicas, as quais podem vir a beneficiar esse determinado público. Portanto, esse trabalho pode contribuir informando a comunidade acadêmica e a sociedade em geral sobre o acesso dos pacientes a esses recursos. Embora ainda exista mitos, tabus e desafios em torno da saúde mental, é preciso descontruir os estigmas e assim promover ações de prevenção e conscientização sobre a importância do tratamento da depressão.
Acerca das contribuições da pesquisa ao campo de atuação psicológico, é evidente que a Psicologia enquanto ciência interdisciplinar se propõe a discutir temas emergentes, compartilhar experiências exitosas e refletir sobre questões de saúde pública, como é o caso, o encontro de diferentes saberes traz consigo contribuições valiosas para a comunidade apontando melhorias no bem-estar e na qualidade de vida do indivíduo. Quanto as principais limitações deste estudo constatado pela autora encontram-se no sentido de obter materiais pertinentes a pesquisa e de avaliá-los sobre outro viés que não o psicológico.

\section{Referências}

Abreu, P. R., \& Santos, C. (2008). Behavioral models of depression: A critique of the emphasis on positive reinforcement. International Journal of Behavioral and Consultation Therapy, 4(2) 130-145. doi: $10.1037 / \mathrm{h} 0100838$

Andrade, A. F., \& Mello, C. P. C. (2010). Reabilitação ação neuropsicológica na Depressão: um enfoque terapêutico ocupacional. Cadernos Brasileiros de Terapia Ocupacional da UFSCar, 18(1) 49-55. Recuperado de http://www.cadernosdeterapiaocupacional.u fscar.br/index.php/cadernos/article/view/33 $\underline{2}$

American Psychiatric Association Task Force On Electroconvulsive Therapy. (2001). The practice of electroconvulsive therapy: recommendations for treatment, training and privileging. Washington, DC: Author.

American Psychiatry Association. (2014). Manual diagnóstico e estatístico de transtornos mentais (5a ed.). Porto Alegre: Artmed.

Almeida, A. M. de, \& Lotufo Neto, F. (2003). Diretrizes metodológicas para investigar estados alterados de consciência e experiências anômalas. Archives of Clinical Psychiatry (São Paulo), 30(1), 21-28. doi: 10.1590/s0101-60832003000100003

Bahls, S. C., \& Bahls, F. R. C. (2003). Psicoterapias da depressão na infância e na adolescência. Estudos de Psicologia
(Campinas), 20(2), 25-34. doi: 10.1590/s0103-166x2003000200003

Bear, M. F., Connors, B. W., \& Paradiso, M. A. (2017). Neurociências: desvendando o sistema nervoso (4a ed.). Porto Alegre: Artmed.

Beck, A. T., Rush, A. J., Shaw, B. F., \& Emery, G. (1997). Terapia cognitiva da depressão. Porto Alegre: Artes Médicas.

Beck, J. S. (2013). Terapia CognitivaComportamental: teoria e prática (2a ed.). Porto Alegre: Artmed.

Beck, A. T., \& Bredemeier, K. (2016). A Unified Model of Depression. Clinical Psychological Science, 4(4), 596-619. doi: 10.1177/2167702616628523

Bolsoni-Silva, A. T., \& Loureiro, S. R. (2015). Anxiety and depression in brazilian undergraduate students: the role of sociodemographic variables, undergraduate course characteristics and social skills. British Journal of Applied Science \& Technology, 297-307. Recuperado de http://www.sciencedomain.org/reviewhistory/6616

Brent, D., Emslie, G., Clarke, G., Wagner, K. D., Asarnow, J. R., Keller, M., ... Leonard, H. (2008). Switching to Another SSRI or to Venlafaxine With or Without Cognitive Behavioral Therapy for Adolescents With SSRI-Resistant Depression. JAMA, 299(8), 901-913. doi: $\underline{10.1001 / j a m a .299 .8 .901}$ 
Brunoni, A. R. (2012). Tratamento do transtorno depressivo maior com estimulação transcraniana por corrente contínua: Ensaio clínico, aleatorizado, duplo- cego, fatorial (Tese de Doutorado). Universidade de São Paulo, São Paulo, SP, Brasil.

Campos, J. R., Del Prette, A., \& Del Prette, Z. A. P. (2014). Depressão na adolescência: Habilidades sociais e variáveis sociodemográficas como fatores de risco/proteção. Estudos e Pesquisas em Psicologia, 14(2),408-428.

Canadian Network for Mood and Anxiety Treatments [CANMAT]. (2012). Treating depressive disorder: relapse and recurrence. Recuperado de http://www.canmat.org/cme-depressionrelapse-and-recurrence.php

Cardoso, L. R. D. (2011). Psicoterapias comportamentais no tratamento da depressão. Psicologia Argumento, 29(67) 479-489. doi: 10.7213/rpa.v29i67.20359

Carneiro, A. M., \& Dobson, K. S. (2016). Cognitive-behavioral treatment for major depressive disorder: a narrative review. Revista Brasileira de Terapias Cognitivas, 12(1), 42-49. doi: 10.5935/1808-5687.20160007

Cooper, W. O., Callahan, S. T., Shintani, A., Fuchs, D. C., Shelton, R. C. Dudley, J. A., ... Ray, W. A. (2014). Antidepressants and suicide attempts in children. Pediatrics, 133(2) 204-210. doi: 10.1542/peds.20130923

Costa, R. A., Soares, H. L. R., \& Teixeira, J. A. C. (2007). Benefícios da atividade física e do exercício físico na depressão. Revista Do Departamento de Psicologia. UFF, 19(1), 273-274. doi: 10.1590/s0104$\underline{80232007000100022}$

Cuijpers, P. (2016). Are all psychotherapies equally effective in the treatment of adult depression? The lack of statistical power of comparative outcome studies. Evidence Based Mental Health, 19(2), 39-42. doi: 10.1136/eb-2016-102341

Davis, A. K., Barrett, F. S., May, D. G., Cosimano, M. P., Sepeda, N. D., Johnson, M. W., ... Griffiths, R. R. (2021). Effects of Psilocybin-Assisted Therapy on Major

Depressive Disorder. JAMA

Psychiatry, 78(5), 481- 489. doi:

10.1001/jamapsychiatry.2020.3285

Departamento de Informática do Sistema Único de Saúde do Brasil (DATASUS). (2012). Saúde Pública em Alerta: No Brasil, mortes por depressão crescem mais de $700 \%$ em 16 anos mostram dados do DATASUS. Brasil: DATASUS. Recuperado de

http://datasus.saude.gov.br/noticias/atualiza coes/512-saude-publica-em-alerta-nobrasil-mortes-por-depressao-crescem-maisde-700-em-16-anos-mostram-dados-dodatasus

Dobson, K. S. (2016). New frontiers in cognitive-behavioral therapy for depression. International Journal of Cognitive Therapy, 9(2) 107-123. doi:10.1521/ijct.2016.9.2.107

Dunn, T. W., Vittengl, J. R., Clark, L. A., Carmody, T., Thase, M. E., \& Jarrett, R. B. (2011). Change in psychosocial functioning and depressive symptoms during acutephase cognitive therapy for depression. Psychological Medicine, 42(2), 317-326. doi: 10.1017/s0033291711001279

Fava, G. A., Rafanelli, C., Grandi, S., Conti, S., \& Belluardo P. (1998). Prevention of recurrent depression with cognitive behavioral therapy. Arch Gen Psychiatry, 55(9) 816-820. doi: 10.1001/archpsyc.55.9.816

Fernandes, C. S., Falcone, E. M. O., \& Sardinha, A. (2012). Deficiências em habilidades sociais na depressão: Um estudo comparativo. Psicologia: Teoria e Prática, 14(1) 183-196. Recuperado de http://pepsic.bvsalud.org/scielo.php?script= sci_arttext\&pid=S1516$36872012000100014 \& \operatorname{lng}=p t \& \operatorname{lng}=\mathrm{pt}$

Fleck, M. P., Berlim, M. T., Lafer, B., Sougey, E. B., Porto, J. A. D., Brasil, M. A., .. . Hetem, L. A. (2009). Revisão das diretrizes da Associação Médica Brasileira para o tratamento da depressão (Versão integral). Brazilian Journal of Psychiatry, 
31(Suppl. 1), S7-S17. doi: 10.1590/S151644462009000500003

Freire, T. F. V. (2016). Associação de eletroconvulsoterapia a tratamento farmacológico no transtorno depressivo maior: análise de desfecho clínico, marcadores inflamatórios e neurotrofinas (Tese de Doutorado). Faculdade de medicina programa de pós-graduação em psiquiatria e ciências do comportamento, Universidade Federal do Rio Grande do Sul, Porto Alegre, RS, Brasil.

Friedman, E. S., Thase, M. E., Wisniewski, S. R., Trivedi, M. H., Biggs, M. M., Fava, M., ... Rush, A. J. (2009). Cognitive therapy argumentation versus CT switch treatment: A STAR*D report. International Journal of Cognitive Therapy, 2(1) 66-87.

Goodier, R. (2014). Is depression less common among men, or are they less likely to acknowledge it? Nova York: Medscape.

Gomes, I. S., \& Caminha, I. D. O. (2014). Guia para estudos de revisão sistemática: uma opção metodológica para as Ciências do Movimento Humano. Movimento, 20(1) 395-411. Recuperado de https://pesquisa.bvsalud.org/portal/resource /pt/biblio-995769

Gottlieb, J. D., Mueser, K. T., Rosenberg, S. D., Xie, H., \& Wolfe, R. S. (2011).

Psychotic Depression, Posttraumatic Stress Disorder, and Engagement in CognitiveBehavioral Therapy within an Outpatient Sample of Adults with Serious Mental Illness. Comprehensive Psychiatry, 52(1) 41-49. doi: 10.1016/j.comppsych.2010.04.012

Hofmann S. G. (2014). Introdução à terapia cognitivo-comportamental contemporânea. Porto Alegre: Artmed.

Lefaucheur, J.-P., André-Obadia, N., Antal, A., Ayache, S. S., Baeken, C., Benninger, D. H., ... Oliviero, A. (2014). Evidencebased guidelines on the therapeutic use of repetitive transcranial magnetic stimulation (rTMS). Clinical Neurophysiology, 125(11), 2150-2206. doi: 10.1016/j.clinph.2014.05.021

Kobayashi, M., \& Pascual-Leone, A. (2003). Transcranial magnetic stimulation in neurology. The Lancet Neurology, 2(3), 145-156. doi:

https://doi.org/10.1016/s14744422(03)00321-1

Koenig, M., \& Thase, M. E. (2009). First -line pharmacotherapies for depression: What is the best choice? Polskie Archiwum Medycyny Wewnetrznej, 119(7-8) 478-486.

Matos, A. C. S., \& Oliveira, I. R. (2013). Terapia cognitivo-comportamental da depressão: Relato de caso. Revista de Ciências Médicas e Biológicas, 12(4), 512519.

Mclntyre, R. S. (2015). Clínico geral, psiquiatra, psicólogo, paciente. A quadratura do círculo? Disfunção cognitiva no transtorno depressivo maior. Canadá: Medscape Education. Recuperado de

http://img.medscapestatic.com/images/846/ 378/846378_webreprint_por.pdf

Moffa, A. H. D. M. (2016). Estimulação transcraniana por corrente contínua na fase aguda do episódio depressivo maior: Uma meta-análise de dados individuais (Dissertação de Mestrado). Instituto de Psicologia na Universidade de São Paulo, SP, Brasil.

Montenegro, M. C., \& Cantilino, A. (2016). Estimulação magnética transcraniana: o que o psiquiatra deve saber?. Debates Em Psiquiatria, 6(3), 23-36. Recuperado de https://www.revistardp.org.br/revista/article /view/131

Moser, C. M., Lobato, M. I., \& Belmonte-DeAbreu, P. (2005). Evidências da eficácia da eletroconvulsoterapia na prática psiquiátrica. Revista de Psiquiatria do Rio Grande do Sul, 27(3) 302-310. doi: 10.1590/S0101-81082005000300009

Oliveira, P. A. (2010). Habilidades sociais, depressão, ansiedade e alcoolismo em bombeiros: Um estudo correlacional (Dissertação de Mestrado). Universidade Federal de São Carlos, São Carlos, SP, Brasil.

Pastore, D. L., Bruno, L. M., Nardi, A. E., \& Dias, A. G. (2008). O uso da eletroconvulsoterapia no Instituto de Psiquiatria da Universidade Federal do Rio 
de Janeiro no período de 2005 a 2007. Revista de Psiquiatria do Rio Grande do Sul, 30(3), 175-181. doi: 10.1590/S010181082008000400006

Powell, V. B., Abreu, N., Oliveira, I. R., \& Sudak, D. (2008). Terapia cognitivocomportamental da depressão. Brazilian Journal of Psychiatry, 30(Suppl. 2), 73-80. doi: $10.1590 /$ S1516-44462008000600004

Ribeiro, R. B., Melzer-Ribeiro, D. L., Rigonatti, S. P., \& Cordeiro, Q. (2012). Electroconvulsive therapy in Brazil after the "psychiatric reform": a public health problem-example from a university service. J ECT., 28(3) 170-173. doi:10.1097/YCT.0b013e31824d2889

Rothschild, A. J. (2013). Challenges in the Treatment of Major Depressive Disorder with Psychotic Features. Schizophrenia Bulletin, 39(3) 787-796. doi: $10.1093 / \mathrm{schbul} / \mathrm{sbt} 046$

Solomon, A. (2014). O demônio do meio dia: Uma anatomia da depressão (2a ed.). São Paulo: Companhia das letras.

Stahl, S. M. (2014). Psicofarmacologia: bases neurocientíficas e aplicações práticas $(4 \mathrm{a}$ ed.). Rio de Janeiro: Guanabara Koogan.

Stopa, S. R., Malta, D. C., Oliveira, M. M. de, Lopes, C. de S., Menezes, P. R., \& Kinoshita, R. T. (2015). Prevalência do autorrelato de depressão no Brasil: resultados da Pesquisa Nacional de Saúde, 2013. Revista Brasileira de Epidemiologia, 18(suppl 2), 170-180. doi: 10.1590/1980-5497201500060015

Tomas, A. C. T., \& Carvalho, M. R. (2014). Treino assertivo para a depressão: uma revisão bibliográfica. Revista Brasileira de
Terapias Cognitivas, 10(2), 103-111. doi: 10.5935/1808-5687.20140016

Vosgerau, D. S. A. R., \& Romanawski, J. P. (2014). Estudos de revisão: implicações conceituais e metodológicas. Revista de Diálogo Educacional, 14(41) 165-189. Recuperado de https://periodicos.pucpr.br/index.php/dialog oeducacional/article/view/2317

Wenzel, A., Brown, G. K., \& Beck, A. T. (2010). Terapia cognitivo-comportamental para pacientes suicidas. Porto Alegre: Artmed.

Wiles, N., Thomas, L., Abel, A., Barnes, M., Carroll, F., Ridgway, N., ... Turner, K. (2014). Clinical effectiveness and costeffectiveness of cognitive behavioural therapy as an adjunct to pharmacotherapy for treatment-resistant depression in primary care: the CoBalT randomised controlled trial. Health Technology Assessment, 18(31), 1-167. doi: 10.3310/hta18310

World Health Organization. (WHO). (2009). Mental health: new unders tanding, new hope. Genova: WHO.

World Health Organization (WHO). (2010). International statistical classification of diseases and related health problems - ICD10 (10th ed.). Genova: WHO.

World Health Organization. (WHO). (2013). Mental Health Action Plan 2013-2020. Geneva: Author.

World Health Organization. (WHO). (2016). World health statistics 2016: monitoring health for the SDGs, sustainable development goals. Geneva: WHO. 


\section{Dados sobre os autores:}

- Fabiane Schott: Mestranda em Ciências da Saúde-UFSM- Especialista em Psicologia Jurídica e Avaliação Psicológica pela Faculdade Dom Alberto; Bacharel em Psicologia pela Faculdade Integrada de Santa Maria-FISMA, Pós graduanda em Psicologia Infantil.

- Júlia Luciane Vidal: Residente no Programa de Residência Multiprofissional Integrada em Onco-Hematologia no Sistema Único de Saúde UFSM/HUSM. Bacharel em Psicologia pela Faculdade Integrada de Santa Maria-FISMA. Santa Maria.

- Alice Dias Pasche: Residente no Programa de Residência Multiprofissional Integrada em Saúde Mental no Sistema Único de Saúde UFSM/HUSM. Bacharel em Psicologia pela Faculdade Integrada de Santa Maria-FISMA. Santa Maria.

- Guilherme Corrêa: Mestre em Psicologia da Saúde pela UFSM. Docente da faculdade Integrada de Santa Maria-FISMA. Santa Maria.

\section{Declaração de Direito Autoral}

A submissão de originais para este periódico implica na transferência, pelos autores, dos direitos de publicação impressa e digital. Os direitos autorais para os artigos publicados são do autor, com direitos do periódico sobre a primeira publicação. Os autores somente poderão utilizar os mesmos resultados em outras publicações indicando claramente este periódico como o meio da publicação original. Em virtude de sermos um periódico de acesso aberto, permite-se o uso gratuito dos artigos em aplicações educacionais e científicas desde que citada a fonte conforme a licença CC-BY da Creative Commons. 